\title{
Anthrovision
}

Vaneasa Online Journal

\section{Cinéma amérindien brésilien et utilisation du cyberspace. Pour qui?}

Paula Morgado

\section{(2) OpenEdition \\ 1 Journals}

\section{Electronic version}

URL: http://journals.openedition.org/anthrovision/1448

DOI: 10.4000/anthrovision. 1448

ISSN: 2198-6754

\section{Publisher}

VANEASA - Visual Anthropology Network of European Association of Social Anthropologists

\section{Electronic reference}

Paula Morgado, "Cinéma amérindien brésilien et utilisation du cyberspace. Pour qui? », Anthrovision [Online], 2.2 | 2014, Online since 05 January 2015, connection on 22 September 2020. URL : http:// journals.openedition.org/anthrovision/1448; DOI : https://doi.org/10.4000/anthrovision.1448

This text was automatically generated on 22 September 2020 .

(C) Anthrovision 


\title{
Cinéma amérindien brésilien et utilisation du cyberspace. Pour qui?
}

\author{
Paula Morgado
}

\begin{abstract}
Remerciement
Je tiens à remercier Barbara Glowczewski et Florence Brunois qui ont organisé le Colloque L'envers du Décor - Émergence des formes et agencements d'existence (tenu au Collège de France du 29 au 31 janvier 2014) pour lequel cet article a été conçu. Je remercie également la FAPESP (Fundação de Amparo à Pesquisa do Estado de São Paulo) qui m'a permis de me rendre à Paris, comme chercheur, de novembre 2013 à mars 2014, Nadine Wanono et son laboratoire l'Institut des Mondes Africains et qui m'a accueilli pendant ce séjour, les évaluateurs de cette revue, dont les commentaires ont été précieux pour la finalisation du présent texte et également Sylvie Gradel qui m'a aidé dans la formulation du premier manuscrit en français Une liste d'URL contenant les portails, sites web, blogs et sites de références à la base de la construction de ce texte sont indiqués à la fin.
\end{abstract}

\section{Introduction}

1 Mon premier terrain chez les amérindiens s'est réalisé en Amazonie durant une période pré-Internet (1989-2002), lorsque la vidéo, la télé et plus tard, le mobile, représentaient pour les amérindiens de nouvelles technologies. Puis en 2006, j'ai commencé à travailler dans le Nord-Est du Brésil avec des communautés qui depuis 4 siècles essaient obstinément de survivre, même si elles gardent un contact étroit avec la société occidentale qui les entoure. C'est dans ces communautés du Nordeste qu'Internet a pu se développer de manière plus systématique, tandis que la vidéo l'a été davantage parmi les populations amazoniennes - qui concentrent plus de la moitié des Indiens du pays. Ces phénomènes médiatiques ne se sont pas produits par hasard au Brésil. Si, d'une part, les groupes du Nordeste ont en premier ressenti plus fortement l'impact de la proximité des coutumes occidentales sur leurs modes de vie, ils ont d'autre part aussi pu profiter davantage des politiques d'inclusion numérique mises en place par le gouvernement. Les groupes amazoniens, demeurés pluslongtemps isolés de la 
société occidentale moderne, ont été la cible de multiples projets filmiques, comme nous le verrons.

2 En 1990, à l'occasion des commémorations des 500 ans de l'Amérique, j'ai pu suivre un débat passionnant lors du festival: «L'indien : hier, aujourd'hui et demain $»^{1}$. Certains amérindiens commençaient alors à s'approprier la vidéo et faisaient de ce support un instrument permettant d'enregistrer et de préserver leur culture. Pour les anthropologues et tous ceux qui se consacraient depuis longtemps à l'étude des cultures autochtones, il devenait évident que la vidéo était un formidable moyen pour comprendre, dialoguer et faire partager la diversité des "peuples indigènes » (ou «povos indígenas»), le nom par lequel on désigne les Indiens au Brésil et qu'ils ont eux-mêmes adoptés.

3 Contrairement aux pronostiques négatifs circulant jusqu'au milieu des années 70 qui annonçaient la disparition inévitable de ces peuples indigènes du Brésil, la production cinématographique amérindienne de ces 20 dernières années prouve que loin d'être absorbées par la société nationale, ces populations ont su s'imposer et lutter pour préserver, par ce biais, leur diversité culturelle ${ }^{2}$.

4 De la fin des années 80 à nos jours, l'intérêt des amérindiens pour la production de films a sensiblement augmenté, et dans diverses régions du Brésil de jeunes cinéastes se forment à ces techniques. Peu à peu, ils surmontent la marginalisation sociale locale et le regard hautement suspect des anciens de leurs communautés, pour conquérir un statut dans celles-ci, assumant un rôle culturel et politique important dans le dialogue interculturel: entre leurs communautés, entre celles-ci et le mouvement panamérindien, et avec la société nationale brésilienne et internationale.

5 Au cours de l'histoire des amérindiens - et ce de façon marquante dans les Amériques au travers de leurs luttes contre la société occidentale moderne, nous avons assisté à l'avènement d'une élite à plusieurs facettes : dans les arts - des peintres amérindiens originaires des sociétés aztèques et mayas du Mexique qui fréquentaient les cours du XVIIème et XVIIIème siècles, jusqu'aux artistes du XXIème siècle dont les travaux sont exposés dans les grandes galeries et les musées; en politique, avec l'apparition de leaders, façonnés par des institutions extra-locales et qui se consacrent aux enjeux de politique nationale; dans l'économie, où ils participent à de grands projets nationaux ou locaux dans le domaine du tourisme; dans l'éducation, en formant de nouveaux universitaires, donc une élite amérindienne dotée de compétences nouvelles. Récemment, certains d'entre eux se sont appropriés des outils médiatiques, comme la vidéo et le web dans leur lutte pour la différence. Il s'agit de personnes qui transitent entre différents mondes, se rapprochant de nous, anthropologues, en tant qu'interprètes ou traducteurs culturels.

6 Néanmoins, même si nous observons une production accrue de la part de ces nouveaux leaders - universitaires, professionnels libéraux, écrivains, cinéastes, blogueurs -, les peuples amérindiens du Brésil n'en demeurent pas moins confrontés à toutes sortes de préjugés de la part d'une grande partie de la société brésilienne. Parfois traités avec dédain ou mépris, parfois luttant pour un espace sur le territoire national: pour certains c'est une question de survie physique, pour tous il s'agit d'une question d'acceptation culturelle. Nous avons bien affaire ici à un processus marqué par des luttes, des injustices et, bien souvent, par une silencieuse extermination culturelle, présente dans toute l'Amérique. 
7 Pour appréhender la diversité et la complexité de la réalité indigène au Brésil, il ne faut pas oublier certains faits. Il existe 180 langues indigènes parlées, réparties entre 305 peuples différentes, dont 43 résidant dans des régions frontalières du Brésil. C'est seulement à partir de 1991 que les indigènes ont été incorporés pour la première fois au recensement national ${ }^{3}$, mais, malgré cela, nous ne connaissons pas avec précision leur nombre. D'après le dernier recensement de 2010, la population comptait environs 896 000 indigènes, dans 24 des 27 états brésiliens, $46 \%$ de la population indigène dans la région Nord et $36 \%$ dans les villes et plus de 300000 personnes vivent en milieu rural. La population entière ne représente pas plus de $0,50 \%$ de la population totale du pays.

8 Le Brésil, de par ses dimensions continentales, abrite des populations indigènes qui maintiennent des contacts sporadiques, intermittents ou permanents avec la société nationale, selon la région où elles se trouvent. Si certains peuples ont survécu à un contact ancien, depuis les premières rencontres avec les Européens, au XVIIème siècle, une majorité d'entre eux n'a commencé à avoir de contacts que dans les années 1970, certains peuples n'ayant été que très peu contactés par les acteurs économiques ou s'en étant tenus à distance. Dans le domaine de l'enseignement formel brésilien, il faut bien dire que, de manière générale, le thème indigène est malheureusement très mal abordé et que le brésilien commun sait peu de choses sur l'histoire et l'héritage culturel indigène ${ }^{4 .}$ Néanmoins, la langue brésilienne s'alimente fortement de mots indigènes; sa cuisine possède des racines indigènes; l'architecture des "caiçaras", peuples habitant la zone littorale, ou des petits agriculteurs de l'intérieur des terres, conserve une énorme similitude avec celle de beaucoup de peuples indigènes. Nous assistons à la divulgation systématique, dans les grands médias, de conflits de tous ordres sur des réalités indigènes distinctes. La publication d'innombrables études, des expositions et des festivals de cinéma touchant à cette réalité envahissent les villes. Malgré tout cela, les peuples indigènes occupent encore un espace marginal dans la société brésilienne et ce profond hiatus est loin de disparaître.

9 Face à la criante méconnaissance brésilienne et à une réalité amérindienne plurielle, le cinéma produit sur cette dernière a été extrêmement idéalisé ou imaginé. Il s'agit d'images qui, parlant de ces peuples, en disent plus long sur le regard de ceux qui se trouvent derrière les caméras que sur les protagonistes, révélant les dilemmes d'un Brésil diversifié qui sait peu de choses sur lui-même. En 2000, dans le contexte des commémorations des 500 ans de la découverte du Brésil, j'étais curatrice du Festival Les Brésils Autochtones avec l'intention de dépeindre cet imaginaire et, en même temps, de célébrer l'avènement d'une filmographie amérindienne propre. A l'occasion de ce festival, nous avons montré que non seulement ces peuples continuaient à inspirer les cinéastes et les anthropologues pour la production de documentaires et de films de fiction, mais qu'ils commençaient à réaliser leurs propres films. Ils ne voulaient plus être traités d'INDIENS, mais de Kayapo, Xavante, Suya, Wayana, Caxinawa, Yanomami, Guarani, Pankararu, et ainsi de suite.

Des films comme "Wapté Mnôhnõ, initiation du jeune Xavante» (1999) (http:// www.videonasaldeias.org.br/2009/video.php?c=65), réalisé par quatre indiens Xavante et un indien Suyá, ont mis en évidence l'intérêt du grand public et des groupes indigènes eux-mêmes qui souhaitent s'égaler aux autres professionnels de l'industrie cinématographique. Récemment, le film " Les hyperfemmes (http:// www.videonasaldeias.org.br/2009/video.php?c=116) (2011) -qui résulte d'un partenariat entre un cinéaste indigène Kuikuro, un anthropologue et un cinéaste 
brésilien - a vu sa consécration lors de deux importants festivals brésiliens et il a ainsi inauguré une nouvelle phase du cinéma indigène brésilien. Bien au-delà du partenariat entre professionnels de l'image et membres de la communauté indigène, et de l'intérêt des différents protagonistes pour la réalisation d'un produit audiovisuel de qualité, on n'assiste pas seulement à l'émergence d'un public sensible à la question indigène, mais à l'insertion du cinéma au sein des sociétés locales, au service de leurs manifestations culturelles. Grâce aux technologies cinématographiques des Blancs « la fête revient au cinéma " (Migliorin 2013 :276) et tout ce qui est en jeu dans la tradition de la fête Jamunkumalu - hyperfemmes, en langue kuikuro. En d'autres mots, pour que la fête se réalise, il est nécessaire de satisfaire des besoins matériels et parmi ces moyens il est question d'instrumentaliser la présence des Blancs dans le village. Et c'est grâce au cinéma que la tradition se réalise.

10 "Ces passages [du film], les vas-et-vient entre la vie inventée et la vie vécue, produisent le ton du film et seront essentiels pour aboutir à la fête - paradigme de ce mélange entre la performance et la vie quotidienne, entre la mémoire et la fabulation, la cosmologie à l'actualité. La fête n'est pas quelque chose qui existe et qui sera enregistrée, mais qui se produira tout au long du film. On y voit des successions de

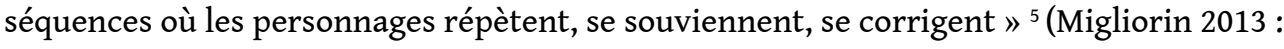
282).

11 Mais qu'est-ce qui amène les peuples indigènes à se sentir si à l'aise face à la caméra ou derrière celle-ci? Les échanges culturels motivés par l'introduction de la vidéo et d'autres outils médiatiques démontrent que l'interaction entre les groupes est moins liée à une demande de "sauvetage » des traditions qu'à une politique d'affrontement plus efficace face aux mondes des «Blancs». Mais les films sont-ils seulement des instruments culturels et politiques? Est-il pertinent de nous interroger sur le fait qu'il existe un cinéma indigène brésilien? Pour qui, cette question a-t-elle un sens ontologique?

12 L'internet a permis la décentralisation de la diffusion des connaissances et leur meilleure distribution. Avec la naissance du WEB, nous assistons à l'avènement d'une immense potentialisation des processus coopératifs au sein des économies décentralisées et le cinéma amérindien brésilien n'échappe pas à cette tendance. Mais, du point de vue de la perspective des peuples amérindiens, observons-nous des contraintes similaires ou distinctes dans le passage d'un media à l'autre, c'est-à-dire, de la vidéo aux nouveaux outils médiatiques?

\section{Les premiers pas du cinéma indigène autochtone}

13 Les premières expériences vidéo, parmi les populations indigènes du Brésil, ont commencé à se développer, de façon systématique, dans la deuxième partie des années 80 , grâce à l'initiative d'ONG en partenariat avec des anthropologues et des universités. Parmi elles, je citerai entre autres : l'expérience pionnière parmi les Kayapo, en 1985, avec le projet de média indigène brésilien intitulé «Mekaron Opoi D'joi » ${ }^{6}$; parmi les Kaxinauá du Acre, dans le nord-ouest de l'Amazonie, à la fin de la même décade, dans un contexte de projets d'éducation différenciée en territoire indigène et peu à peu, d'autres groupes ont pu avoir accès aux outils audiovisuels. L'initiative la mieux réussie a certainement été le projet Vidéo dans les Villages, (http:// www.videonasaldeias.org.br/2009/vna.php?p=1) créé en 1987 à l'initiative de l'ONG 
Centre de Travail Indigéniste qui réunissait depuis 1979 des anthropologues, des éducateurs et des indigénistes. Profitant d'un travail politique dans les communautés indigènes, ils commencèrent à promouvoir des activités de sensibilisation à l'audiovisuel et de façon systématique à organiser les premiers ateliers de formation audiovisuelle pour des membres indigènes.

Peu à peu la caméra offre à ces jeunes réalisateurs un nouveau rôle social. Ils deviennent médiateurs d'un processus de communication englobant divers agents : 1 . la communication qui s'établit au travers de la vidéo entre différents villages d'un même groupe ; 2 la communication entre les différentes générations, vieux, jeunes et enfants et la certitude que les traditions pourront être maintenues; 3 . la communication entre les membres indigènes de différentes sociétés (au Brésil et à l'étranger), qui, par le biais de la vidéo, perçoivent immédiatement que, malgré les différences (et les similitudes véhiculées de fait par la vidéo), ils partagent les mêmes conditions, ce qui peut amener à les fortifier en tant que segment social; 4. la communication entre les leaders et leurs communautés, puisque la vidéo permet de filmer les réunions organisées, par exemple avec les autorités de Fundação Nacional do Índio (FUNAI) et montrer ce qui peut être demandé à cet organisme.

$\mathrm{Au}$ niveau des sujets choisis par les réalisateurs amérindiens, on assiste à trois mouvements chronologiques, mais qui s'entrecoupent par la suite: des films qui abordent les cérémonies, en général les rites d'initiation, qui valident l'ethos du peuple et agissent comme des marqueurs d'altérité ; des films qui abordent le quotidien et qui, petit à petit, gagnent une saveur d'intimité - ils exploitent soient des aspects de la vie de tous les jours, soient des sujets sensibles et des questions conflictuelles pour dénoncer des réalités; des films de fiction qui mettent en scène des mythes. Il est intéressant de noter la maîtrise, de plus en plus présente, du caractère performatif de soi derrière ces productions. Les jeunes réalisateurs autochtones sont à la fois derrières et devant les caméras, car en plus de les enregistrer ils participent aussi aux scènes filmées. Et petit à petit, tout le village joue le jeu de la mise-en-scène cinématographique et on ne distingue plus les scènes capturées par les caméras et celles qui ont été fabriquées pour les gens impliquées lors du tournage ${ }^{7}$.

Cela n'a pas été (et ce n'est toujours pas) sans difficulté que les jeunes se sont imposés dans leurs communautés en leur qualité de cinéastes. Les anciens, comme dans toute société, se plaignent que les plus jeunes ne s'intéressent plus à leurs traditions et ont peur de diffuser leur savoir à l'extérieur de leurs communautés. Mais peu à peu, par un dialogue interne, progressivement mûrit entre les générations, la reconnaissance de l'enregistrement audiovisuel joue en faveur d'une revitalisation culturelle revendiquée. C'est ce dialogue turbulent intergénérationnel qui est au centre du débat pour les groupes aujourd'hui, d'autant plus qu'internet induit une rapidité de la circulation des connaissances que la vidéo n'imposait pas.

Quand nous regardons cette production indigène initiale, certains éléments sont récurrents. La culture est motif de fierté pour ceux qui en parlent. Ce qui est mis en avant est l'emphase sur la tradition, l'importance de ce que les plus âgés transmettent aux plus jeunes, la valorisation de la possibilité de faire quelque chose comme les anciens le faisaient et dans ce sens, la responsabilité des adultes envers les enfants. Dans l'autoreprésentation de ces sociétés, il y a comme un continuum entre les anciens et les jeunes et qu'ils forment un tout. 
18 Aussi, l'introduction de la vidéo dans ces communautés a entraîné un processus de réflexion sur l'image, faisant en sorte que, pour la première fois, les Amérindiens sont à la fois sujets et objets de cette réflexion. Jusqu'alors, malgré la complicité existante entre les communautés indigènes, pro-indigènes et les chercheurs, ceci n'était pas possible, que ce soit dans le domaine de la santé, de l'éducation, du développement culturel etc. Dans ce type de projets, les connaissances extérieures prenaient toujours le pas sur les savoirs locaux et très souvent, ces connaissances étaient mal comprises ou inintéressantes pour les peuples indigènes, du fait de la complexité des moyens de communication qui privilégiaient l'écrit ou l'oralité.

En allant à l'opposé des images qui les transforment en spécimens exotiques, est apparu en 1996 le «Programme d'Indien »,

(http://www.programadeindio.org/index.php?s=pi\&n=pi_historia)

une série composée de quatre émissions de vingt minutes chacune, en partenariat avec la TV Universitaire du Mato Grosso (UFMT), expérience inédite à la télévision brésilienne: l'ouverture d'un espace véritablement indigène sur une chaine publique. Les élèves du projet participaient activement à l'élaboration et à la production des émissions, prenant contact, pour la première fois avec le langage télévisuel. Mais ce projet n'a pas duré longtemps. Treize ans plus tard, entre 2009 et 2011, Vidéo dans les Villages a produit la série «Indiens du Brésil », et, grâce à Internet, celle-ci est disponible en ligne:

http://www.videonasaldeias.org.br/2009/indios_no_brasil.php

23 Mais attention, en général il est encore rare de pouvoir visionner les films dans leur intégralité et lorsque cela est possible, on choisit de les mettre en basse qualité ou seulement un extrait. La méthode de mise en ligne résulte du contrôle des images par les communautés: les connaissances ne peuvent circuler librement, sans le consentement des ânés. À partir du moment où Internet s'impose, les produits audiovisuels commencent à circuler sans le contrôle de la communauté. En outre, il n'est pas rare de trouver les films amérindiens dans leur intégralité hébergés sur d'autres sites Web.

Pour comprendre le contrôle de la circulation, dans une perspective autochtone, il faut considérer que bien que leurs films entrent dans un circuit peu significatif au niveau commercial, les communautés ont l'espoir que ces films, en tant qu'objets (DVDs, Blueray etc) puissent leurs apporter des compensations financières. D'autre part, même si les films abordent des questions du quotidien, le fait de ne pas les rendre accessibles sur internet est un moyen de maintenir un plus grand contrôle sur leur circulation. Aussi, on ne peut pas d'un moment à l'autre retirer l'aura symbolique de l'objet-film. Si le savoir numérique déclenche un débat important parmi les sociétés qui produisent librement du savoir et des outils, qu'est-ce qu'on peut dire de sociétés où la matérialisation de l'immatériel, par l'image, joue un rôle si décisif?

\section{Barrières transposées, transformations et tendances indigènes.}

25 Le cinéma amérindien a déjà inspiré de nombreux festivals au cours de ces vingt dernières années et avec les leaders indigènes, les cinéastes indigènes se sont habitués à discuter sur des plateaux, même s'ils y sont encore souvent traités comme s'ils ne 
dominaient pas, ou peu, nos codes. La formation audiovisuelle s'est étendue dans plusieurs territoires indigènes et aujourd'hui certains amérindiens dispensent des formations audiovisuelles à d'autres jeunes, cette démarche s'inscrivant dans le même agenda politique interne.

Chaque jour se créent de nouveaux collectifs indigènes et certaines de leurs productions sont sélectionnées pour des festivals, bien qu'ils organisent leurs propres festivals en partenariat avec des Universités ou ONG. Ces initiatives vont de paire avec celles nées à l'étranger qui diffusent des productions réalisées par des amérindiens, comme celles de l'association française « De la Plume à l'écran », créée en 2008 (http:// www.delaplumealecran.org/\#contact).

Parmi ces collectifs je signalerai : le Collectif Kuikuro de Cinéma (http:// coletivokuikurodecinema.blogspot.com.br/), dont l'un des jeunes réalisateurs, Takumã, a participé au film Les Hyper femmes, déjà cité, qui a connu un succès inattendu dans le milieu cinématographique; le Collectif Pajé Filmes (http://www.pajefilmes.blogspot.com.br/) ${ }^{8}$, dont le film Xupapoynãg (http://www.youtube.com/watch? v=MvNkzdMarRQ\#t=11, 2012), d'Isael Maxakali, a été sélectionné dans plusieurs festivals internationaux et le Collectif Mbya-Guarani de Cinéma, dont le film Vélos de Nhanderu (http://www.youtube.com/watch?v=7UEWibtKt70) a connu un beau succès dans les festivals au Brésil.

Des efforts ont été faits pour lancer des séries indigènes pour la TV, mais elles ont eu la vie courte et n'ont été diffusées que régionalement. Un phénomène semblable s'est produit avec les encyclopédies indigènes, idéalisées pour circuler dans les écoles, les kiosques à journaux et les librairies. Elles ont gagné une vie nouvelle, presque vingt ans plus tard, sur une plateforme web de l'ONG Sócio Ambiental (http:// www.socioambiental.org/pt-br/o-isa/programas/povos-indigenas-no-brasil). Aussi, Taru Andé. La rencontre du ciel et de la Terre', est une série en treize épisodes produite par la chaîne TV Futura (2007), mise en ligne pour faire son download (http:// www.futuratec.org.br/details.php?id=162d49e7ccab29af3e04cf9c6cb7257e207dec2f) qui dépeint la vie, les origines et les coutumes de douze ethnies différentes. D'autres séries, comme celle-ci, ont vu le jour, dans le but d'expliquer l'univers indigène au monde extérieur.

Dans ce processus de médiatisation de la culture indigène, nous avons assisté à l'avènement de films produits par les associations indigènes elles-mêmes à l'occasion de rituels ou de rencontres politiques, comme c'est le cas de l'Association Yanomami Hutukara qui s'attache à dénoncer les conflits sur les territoires indigènes ou a commémorer des conquêtes (http://www.hutukara.org/videos/). Je cite comme exemple la $2^{\text {ème }}$ Rencontre des shamans Yanomami, intégralement filmée par le cinégraphiste yanomami Morzaniel Iramari qui éditera une vidéo sur l'événement dans le cadre des commémorations des 20 ans de Terre Indigène Yanomami. La vidéo est diffusée par Hutukara dans toutes les régions du territoire Yanomami et mise à disposition du public sur le site de Hutukara et sur youtube : http://www.youtube.com/ watch?v=1sfy3VxQ2Ak.

Dans ce contexte, il est possible d'observer la manière dont les groupes de leaders s'adressent à la caméra afin d'essayer de sensibiliser les grands médias sur le droit autochtone. Au Brésil, la question socio-environnementale est à l'origine de nombreux projets, certains cinématographiques et dans certains cas, la difficulté de diffusion des connaissances dans le cadre d'une campagne de sensibilisation peut être contournée 
par le truchement du WEB, comme c'est le cas de films plus anciens sur les Kayapo diffusés, grâce aux sites de partage de vidéo, à travers des liens insérés dans de courts écrits par des chercheurs (SALM 2008).

31 Il est intéressant d'observer que lors de ce processus de médiatisation indigène, des savoirs qui ne circulaient qu'à l'intérieur des groupes (tenus pour secrets), commencent à être divulgués dans un nouveau format, vers l'extérieur, par exemple notamment parmi les Caxinaua avec des films traitant de la consommation de Yahuasca, sorte $d$ hallucinogène assez répandu en Amazonie mais qui était auparavant très rarement montré aux non-autochtone. Ces enregistrements commencent à être disponible en ligne grâce au fait qu'ils sont filmés par les peuples indigènes eux-mêmes et dépassent ainsi les frontières de leurs villages, A ce propos je cite le film os cantos do cipó (les chants de la liane) réalisé en 2006, de Tadeu Siã Kaxinawa et Josias Maná Kaxinawa.

D'un côté, nous assistons à la prolifération de webfilms divulgués sur des portails ou des blogs, donnant naissance à des webtvs et de l'autre, à la production de films de plus grande envergure (en termes de production, de recherche, de contenu) qui veulent entrer en compétition avec d'autres productions cinématographiques. En ce qui concerne les webtvs, citons le projet TV Navegar du cinéaste Jorge Bodansky qui, grâce à une embarcation mobile, offre aux peuples des berges du fleuve la possibilité de parler de leur quotidien à travers des produits audiovisuels ${ }^{10}$. Ce projet s'inscrit dans une plateforme libre du WEB, nommé Ecovox ${ }^{11}$. A travers des projets d'éducation, de loisir, de tourisme et certains aspects de manifestations culturels de l'Amazonie, cette initiative cherche à fortifier la culture locale - parmi les peuples indigènes sont les lTicuna et les Zuruahã d'Amazonie.

Une lecture à l'emporte pièce et pessimiste de ce processus voit dans la séduction exercée par le monde des Blancs l'immense désintérêt des jeunes indigènes pour leur propre monde. Il est certain que la prolifération de télévisions, d'internet et de logiques économiques externes, comme la multiplication du nombre de salariés dans les communautés indigènes, contribuent en grande partie au rapprochement avec l'univers «des Blancs». Mais ceci est-il nécessairement en désaccord avec la perspective locale ou la coutume de chaque peuple? Penser que de tels changements sont exclusivement dommageables culturellement parlant, revient une fois encore à condamner les sociétés indigènes à l'immuabilité culturelle et à prétendre que ces sociétés n'ont pas entretenu de contacts les unes avec les autres avant l'arrivée des Européens.

34 Comme le dit l'anthropologue Carlos Fausto, dans son commentaire sur les changements dans le monde kuikuro (peuple du Parc du Xingu, Brésil Central, Mato Grosso)

35 'Le ton de lamentation si souvent entendu lorsque l'on évoque les 'pertes de culture' peut être comparé au sentiment d'abandon connu par l'orphelin et qui caractérise le malade qui est sur le point de perdre son monde pour se transformer en un autre type de personne: esprit, animal, mort. Dans cette logique fataliste - qui contamine tant celui qui appartient à la réalité indigène que celui qui y est extérieur - les rituels agissent majoritairement pour éviter que l'on ne soit plus «Indien » et que l'on devienne « Blanc » (FAUSTO 2010:167).

Dans cette logique on assiste, au sein des communautés, durant les deux dernières décennies, à un intérêt pour l'enregistrement sonore et visuel des principaux rituels, musiques, fabrication des objets, visant la "sauvegarde de leur patrimoine culturel". D’une part, il faut insérer ces phénomènes dans le cadre de demandes de collaboration 
faites par les groupes indigènes auprès des ONG ; d'autre part, il faut se débarrasser du piège de l'opposition tradition/invention, authenticité/invention, communément associée à ces phénomènes et les insérer dans le cadre de la matérialisation de l'immatériel au lieu de reproduire le concept de l'objectivation de la culture. Sans cela, on ne saisira jamais la dynamique immanente de la production de la différence culturelle.

Quand on réfléchit aux nouveaux modes de communications chez les amérindiens, nous devons repenser cette obsession à vouloir qualifier d' "autochtone » ce qui pour eux n'est pas une préoccupation ontologique, mais le résultat d'un processus d'affrontement politique avec la société alentour. Comme je l'ai déjà dit, ce n'est pas eux qui sont venus frapper à notre porte pour apprendre à manipuler des caméras vidéo et récemment à maitriser les outils informatiques. Les films réalisés par des amérindiens sont le fruit d'un long mouvement d'ethnicité: sous le prisme des amérindiens, il s'agit d'un modelage de l'«autochtonie » à la recherche de ses droits; et dans la perspective de celui qui va à leur rencontre, les films sont un puissant moyen de communication pour mieux les comprendre. Nous ne pouvons pas perdre de vue que ce sont des sociétés orales à l'origine, dans lesquelles les gestes et les paroles ont un rôle prépondérant, comme dans un film. L'avantage, donc, pour un tel moyen médiatique est une voie à double sens.

Dans un premier temps, à la fin des années 1970, la question qui se posait au Brésil pour les anthropologues et les défenseurs de la cause indigène était de donner de la visibilité à des peuples qui, jusque-là, étaient inconnus de la majorité de la population et que l'on croyait « condamnés à disparaître ». De fait, au Brésil, cette période a été marquée par un grand essor économique et une découverte des peuples autochtones qui occupaient le no man's land amazonien. Au cours de ce processus, certains peuples ont été décimés et d'autres ont subi une réduction drastique de leur population. Les premiers films réalisés en partenariat avec des membres indigènes dénonçaient cette situation, abordant toutes sortes de conflits (agraires, d'extraction de minerais etc.). D'autres exploraient les signes diacritiques indigènes, pour marquer leur différence avec nous, comme les rituels. Progressivement le quotidien a commencé à être décrit et exploré dans les films et peu après la fiction s'est invitée, se mélangeant au documentaire, avec la mise en scène de thèmes mythiques. Parmi ce genre narratif filmé je souhaite attirer l'attention sur les films : Segredos da Mata de Dominique Gallois et Vicent Carelli (1998, 37 min.); Moyngo, o sonho de Maragareum, de Natuyu, Karané et Kumaré Ikpeng (2002, 44 min.); Nguné Elu, o dia em que a lua menstruou de Takumã Kuikuro et Maricá Kuikuro (2004, 28 min.), Porcos raivosos de Isabel Penoni et Leonardo Sette (10 min, 2012). Grâce à une approche multidisciplinaire entre travail ethnographique et esthétique (avec la vidéo, la photo et le théâtre) ces films ont pu être produits dans le cadre du projet Video dans le Village; les deux premiers films sont issus d'ateliers de vidéo et un atelier de théâtre a permis la réalisation du troisième.

39 A la différence d'autres pays, où la production des connaissances anthropologiques est séparée d'une pratique politique, au Brésil les deux vont de paire. Les anthropologues qui s'impliquent avec leurs interlocuteurs, sont amenés à donner leur opinion sur les projets, à jouer le rôle de conseils, à animer des ateliers de portugais, de notions de santé, d'écologie et d'audiovisuel - un processus semblable se développe en Australie et au Québec depuis de nombreuses années ${ }^{12 .}$ Le cinéma joue sans aucun doute un rôle 
mnémonique de poids pour les groupes chez lesquels la transmission orale jouait exclusivement ce rôle et qui aujourd'hui voient leur mode de transmission menacé.

\section{Dernières considérations - en guise de conclusion}

Pour l'instant - alors que la scène médiatique change très vite pour les groupes minoritaires et pour nous tous - l'appropriation des nouveaux outils de communication présents sur Internet, semblent remplir le même rôle politique joué, il y a vingt, par la vidéo. De même, au niveau technique, des difficultés similaires s'imposent. Pour créer des blogs, des sites, participer à des portails etc., les individus issus des communautés amérindiennes réclament une formation continue et sont confrontés à des problèmes techniques (comme toutes les communautés éloignées des centres urbains, particulièrement au Brésil où il y a un écart visible entre le monde des grandes villes et des villages). Grâce à des projets soutenus par le gouvernement ou les ONG, des ordinateurs parviennent aux villages éloignés, des ateliers de formation y sont organisés; pourtant sans une bonne connexion internet et sans une formation continue à long terme, tout cet investissement s'écroule très vite.

41 En réfléchissant à la transformation de ces outils en moyen de communication -en analysant les contenus et leur mise en page- on en revient au problème de la gestion de la diffusion des savoirs à l'intérieur des groupes. L'Internet, en tant qu'espace par excellence du no man's land, exacerbe la question de la gestion de la circulation des savoirs. Dans les sociétés amérindiennes, ce qui est en jeu c'est le rapport de forces entre aînés et plus jeunes dans la gestion du social et de ce qui peut être diffusé pour le grand public ou ne le peut pas. Dans ce sens la question intergénérationnelle est l'une des questions les plus sensibles lorsque l'on travaille avec les outils audiovisuels. Pour les sociétés non-occidentales, les enjeux concernant la liberté d'expression individuelle (et non plus collective) se présentent comme un nouveau défi.

Réaliser une étude sur les web-autoreprésentations dans la perspective amérindienne, c'est prendre en compte certains a priori :

43 1. Ne pas oublier que dans la lutte pour les droits des autochtones, on s'approprie fréquemment des formes d'expression qui sont traditionnellement extrinsèques ;

44 2. Comprendre la façon de traiter les emprunts culturels consiste à comprendre le contexte dans lequel ces luttes sont nées et à ne pas négliger la pratique d'une bonne ethnographie sur le groupe dans lequel on travaille;

3. Ce n'est pas avec l'avènement de l'Internet que de nombreux groupes ont commencé à matérialiser leurs propres événements culturels à des fins politiques. Au cours des dernières décennies et aujourd'hui, la vidéo a servi d'instrument d'affirmation ethnique, mais les pages web, en plus de remplir la même fonction, ont élargi ce public. Nous ne devons pas perdre de vue les auteurs virtuels hors ligne et leurs univers, afin de comprendre si ces médias sont utilisés pour affirmer des identités locales déjà présentes dans la culture hors-ligne ou si nous observons, comme parmi d'autres communautés virtuelles, la prolifération de nouvelles identités sur Internet. À mon avis il faut prendre en considération, dans cette arène politique, ces deux forces en alternance.

En analysant les discours de représentation amérindiens sur le Web nous devons les inscrire dans le récit critique de la modernité. Les groupes autochtones et les groupes 
auxquels ils se rapportent ne forment pas de mondes séparés (le mélange est structurant pour la construction de leur identité) et les visions qu'ont ces groupes et ceux des autres créent des espaces de communication dont les intersections, les résistances et les mélanges, forment un flux continu d'images dans les deux sens.

Une autre caractéristique à retenir sur les pages Web est l'interaction possible entre les différentes formes d'action, entre le texte, le son et l'image, desquelles émergent les représentations sociales. Dans la plupart des pages Web, à travers des textes et des audiovisuels, on exacerbe les aspects traditionnels (ou ce que chacun entend par "tradition"). Peu importe si ces traditions n'ont plus cours, car pour les auteurs ce qui important est le fait qu'elles puissent agir comme principe éthique.

Compte tenu de l'énorme inégalité entre les forces politiques, hors ou en ligne, les groupes amérindiens finissent par renforcer leurs frontières. Si Internet est perçu comme un monde sans bornes, marquer les territoires parait inefficace. Toutefois les contenus des pages fixent les symboles d'un passé à la place du présent vécu. Dit d'une autre manière, les pages finissent par transmettre des valeurs culturelles susceptibles de renforcer l'esprit d'appartenance à une tradition immuable et elles ne reposent pas sur l'image dynamique que les groupes souhaitent s'attribuer à eux-mêmes. Cela est une des contradictions de ces discours ou le piège du discours visuel est relayé à la puissance maximale par le Web. De même, lorsque l'on se pose la question de savoir " pour qui » est fait le cinéma amérindien brésilien et l'utilisation du cyberspace, la réponse doit être cherchée dans le raisonnement local (autochtone) qui se nourrit des rencontres avec le monde occidental moderne. En d'autres termes, ces deux formes de communication sont choisies par les communautés autochtones à la fois comme nouvel outil de sauvegarde de leur patrimoine matériel et immatériel, mais aussi pour sensibiliser les non-autochtones à leurs luttes pour le droit à la diversité culturelle

Ce qui se passe actuellement dans le domaine du cyberactivisme amérindien nous fait penser à la critique faite, il y a quelques décennies, aux études postcoloniales. Afin de se débarrasser des pratiques associées au colonialisme et au paternalisme, les anthropologues postmodernes ont fini par être critiqués sur leur manque de données ethnographiques. Toutefois, entre le besoin de renouvellement de la pratique et la paralysie de la théorie, ces auteurs sont responsables d'une grande réflexion sur la signification des travaux anthropologiques et je crois que c'est dans cette voie qu'il faut comprendre les premières expériences amérindiennes sur le Web. En termes de structure, leur pages web sont, en grande partie, fondées sur le discours déjà établi par ce type de média, ce qui compte ce sont les objectifs et les rôles joués par les pages web elles-mêmes : les contenus sont au service des mouvements permanents pour le droit à la diversité culturelle. Les pages web amérindiennes doivent être lues dans le cadre du processus de "l'autochtonie " en rapport direct avec les formes d'interventions politiques exercées par le gouvernement sur ces sociétés et comment la société civile y réagit.

50 L'explosion des nouvelles formes de communication et de la technologie (accompagnée par la reconfiguration et la transnationalisation du marché du travail) a permis de surmonter les distances spatiales et temporelles qui, d'un côté ont progressivement changé le scénario de la production des identités et de l'autre, ont généré le sentiment que nous partageons tous une humanité commune. Néanmoins, on n'a jamais eu autant accès à des espaces et à des temps différents qui conduisent à valoriser la diversité. Peu à peu se consolide la possibilité d'expression de voix marginales, elles-mêmes 
reformulées, formant de nouveaux réseaux en faisant partie de ce que l'on appelle les "radical media". Le risque est, à l'extrême, la production de l'exotique, de quelque chose qui n'existe pas et qui est projeté à sa puissance maximale.

51 Si l'anthropologie est destinée à être polyphonique en nous aidant à surmonter les préjugés, le cyberamérindianisme montre que les groupes ne veulent pas rester en dehors de ce mouvement. Et ce qu'on observe à travers les pages web signées par ces derniers, est que les discours sont davantage construits pour sensibiliser les non-autochtones. Ainsi, si avant, les mythes et les rituels jouaient un rôle décisif dans le champs de la transmission du savoir, maintenant les outils médiatiques incorporés sont mis à disposition pour compléter la chaîne de la transmission culturelle pour répondre aux nouveaux besoins politiques engendrés par les rapports avec la société occidentale. Face à la vitesse de changement actuelle, regardons ce que l'avenir nous réserve.

\section{BIBLIOGRAPHY}

\section{List of References}

Brasil, Andre. 2012. Bicicletas de Nhanderu. Lascas do extracampo In Revista Devires. Cinema e Humanidades. Universidade Federal de Minas Gerais. (9), 1: 98-117.

Carelli, Vincent et Gallois, Dominique, dirs. 1998. Segredos da Mata. Vídeo nas Aldeias (production). $37 \mathrm{~min}$.

Coletivo de Kuikuro de Cinema Blog.

http://coletivokuikurodecinema.blogspot.fr/?zX=c5a2321efbf4013b (consulté le 5 Mars, 2014)

Coletivo Pajé filmes Blog.

http://www.paje-filmes.blogspot.com.br (consulté le 5 Mars, 2014)

Fausto, Carlos. 2010 "No registro da Cultura - o cheiro dos brancos e o cinema dos índios" In Vídeo nas Aldeias: 25 anos. Carvalho, Ana et all. (eds.) Pp 160-169. Olinda: Vídeo nas Aldeias, Ed.

Fausto Carlos et Sette, Leonardo, dir, 2011. As hipermulheres. Projeto Video nas Aldeias (production), $80 \mathrm{~min}$.

Frota, M. 1996. Taking Aim: The Video Technology of Cultural Resistance In Resolutions. Contemporary vídeo practices. Renov, Michael and Suderburg, Erika (eds.). Pp.258-282. The University of Minnesota Press.

De la plume à l'écran. 2008. Siteweb

http://www.delaplumealecran.org (consulté le 5 Mars, 2014)

Gallois, Dominique Tilkin. 2011. Materializando saberes imateriais: experiências indígenas na Amazônia Ocidental. Revista de Estudos e Pesquisas (Fundação Nacional do Índio), v. 4, p. p10-20.

Hutukura. 2010 Siteweb 
http://www.hutukara.org/videos/ (consulté le 5 Mars, 2014)

Ikpeng, Karané et all., dirs. 2002. Moyngo, o sonho de Maragareum. Vídeo nas Aldeias (production). $44 \mathrm{~min}$.

Instituto Brasileiro de Geografia e Estatística. Notícias: IBGE mapeia a população indígena. http://cod.ibge.gov.br/1XSTK (consulté le 5 Mars, 2014)

Kaxinawa,Tadeu Siã et Kaxinawa,Josias Maná, dir. 2006. Huni Meka. Os cantos do Cipó. Projeto Video nas Aldeias (production), 26 min.

Kuikuro, Takumã et Kuikuro,Maricá, dirs. 2004. Nguné Elu, o dia em que a lua menstruou. Vídeo nas Aldeias (production). $28 \mathrm{~min}$.

Migliorin, Cezar. Vol.20, 2013. Território e virtualidade: quando a "cultura" retorna no cinema Revista Flamecos. Midia, cultura e tecnologia. 20 (2):275-295. Porto Alegre.

http://revistaseletronicas.pucrs.br/scientiamedica/ojs/index.php/revistafamecos/article/view/ $14207 / 9966$

Ortega,Ariel Duarte and Ferreira,Patricia, dir. 2011.Bicicletas de Nhanderu, Coletivo Mbya-Guarani de Cinema (Production). 48 min.

Ortega,Ariel Duarte and Ferreira,Patricia, dir. 2011. Bicicletas de Nhanderu YouTube. $48 \mathrm{~min}$.

http://www.youtube.com/watch?v=7UEWibtKt70 (Téléchargé par Projeto Abridor 17.08.2012, consulté le 5 Mars, 2014)

Penoni, Isabel et Sette, Leonardo, dirs. 2012. Porcos raivosos. Vídeo nas Aldeias (production). 10 $\min$.

Programa de Indio. 2009. Ikorĕ - projetos culturais e artísticos (production)

http://www.programadeindio.org/index.php?s=pi\&n=pi_historia (consulté le 5 Mars, 2014)

Salm, Rodolfo. Vídeos Kayapó. 2008.

http://www.correiocidadania.com.br/index.php?

option=com_content\&view=article\&id=1513:amcid050308\&catid=28:ambiente-ecidadania\&Itemid=57 (consulté le 5 Mars, 2014)

Tserewahú, Divino, dir 1999. Wapté Mnõhnõ, initiation du jeune Xavante. Video nas Aldeias (Production), $56 \mathrm{~min}$.

Yanomami, Morzaniel. Dir. 2012. lII Encontro de Xamãs Yanomami. YouTube. 3 min 16sec.

http://www.youtube.com/watch?v=1sfy3VxQ2Ak (Téléchargé par SocioAmbiental 07.05.2012, consulté le 5 Mars, 2014)

\section{Webographie}

[consulté le 5 mars, 2014]

\section{Portails}

Índios On-Line

http://www.indiosonline.net/ 
Portal Kaingang

http://www.portalkaingang.org/

Rede Povos da Floresta

www.facebook.com/pages/Rede-Povos-da-Floresta-Fan-Page/224531120943649

Projeto Séculos Indígenas no Brasil

http://www.seculosindigenasnobrasil.com/

Portal Guarani

www.tekoarandu.org

TV Maracá

http://webtvmaraca.wordpress.com

\section{Blogs et sites (de collectifs ou auteurs)}

auteurs amérindiens

Ashaninka (Acre)

www.apiwtxa.blogspot.com

Baniwa (Amazonas)

www.rbaniwa.wordpress.com

www.pamaali.wordpres.com

Guajajara (Maranhão)

http://blogger-aldeiaidgenazutiua.blogspot.fr/

Guarani (São Paulo)

www.oliviojekupe.blogspot.com

Kuikuro (Mato Grosso)

http://coletivokuikurodecinema.blogspot.com.br/

Munduruku (Pará/Paraná)

http://danielmunduruku.blogspot.fr/

Potiguara (Rio de janeiro)

http://www.elianepotiguara.org.br

Tupinambá (Rio de Janeiro)

http://webradiobrasilindigena.wordpress.com/

Tupiniquim - blog sobre povos indígenas

http://indios.blogspot.com/

Yawanawá (Acre)

www.awavena.blog.uol.com.br

Xukuru 
http://www.xukuru.de/

\section{Organisations amériendiennes et de soutien (Brésil)}

Área Indígena

http://www.areaindigena.hpg.ig.com.br/

Adital - Notícias da América Latina e Caribe

http://www.adital.com.br/site/tema.asp?lang=PT\&cod=10

Arte do Mito

http://www.artedomito.com/pag1.htm

Cedefes - Centro de Documentação Eloy Ferreira da Silva

http://www.cedefes.org.br/new/index.php

Centro de Trabalho Indigenista - CTI

http://www.trabalhoindigenista.org.br/

Comissão Pró-Índio do Acre

http://www.cpiacre.org.br/

Comissão Pró-Índio de São Paulo

http://www.cpisp.org.br/

Comissão Pró-Yanomami - CCPY

http://www.proyanomami.org.br/v0904/index.asp

Inbrapi -Instituto Indígena Brasileiro para Propriedade Intelectual

http://www.inbrapi.org.br/

Iepé - Instituto de Pesquisa e Formação em Educação Indígena

http://www.institutoiepe.org.br

Ideti - Instituto das Tradições Indígenas

http://www.ideti.org.br/intro.html

Instituto Socioambiental - ISA

http://www.socioambiental.org

Línguas Indígenas 2000

http://geocities.com/indianlanguages_2000/

OAEYRG/COOPYAWA - Organização de Agricultores e Extrativistas Yawanawá/Cooperativa AgroExtrativista Yawanawá

http://www.yawanawa.com

Operação Amazônia Nativa - Opan

http://www.opan.org.br/

Os Índios Guarani 
http://www.paraty.com.br/guarani/

PESACRE

http://www.pesacre.org.br/qs.htm

PWA - Programa Waimiri Atroari

http://www.waimiriatroari.org.br/

Programa Parakanã

http://www.parakana.org.br

Thidêwá

http://www.thydewa.org/

Vídeo nas Aldeias

http://www.videonasaldeias.org.br

\section{Associations et Conseils de Peuples Amérindiens}

Associação Artístico-Cultural Nhandeva - Nossa Gente

http://www.nhandeva.org/

Anaí - Associação Nacional de Ação Indigenista

http://www.anai.org.br

Apio - Associação dos Povos Indígenas do Oiapoque

http://www.povosindigenasdooiapoque.com.br/

Associação Hutukura Yanomami

http://www.hutukara.org/

Associação Xavante Warã

http://wara.nativeweb.org/index.html

Conselho Indígena de Roraima - CIR

http://www.cir.org.br/

Coordenação das Organizações Indígenas da Amazônia Brasileira - Coiab

http://www.coiab.com.br/

Fotografia e História Junto a Povos Indígenas

http://www.djweb.com.br/historia/

Warã - Instituto Indígena Brasileiro

http://www.institutowara.org.br/

Ponto de Cultura Maloca Digital

http://www.pcmalocadigital.blogspot.fr/ 


\section{Associations catholiques}

Conselho Indigenista Missionário - Cimi

http://www.cimi.org.br/

O Mensageiro

http://freeweb.supereva.com/mensageiro.freeweb/

\section{Sites governamentaux (Brésil)}

Fundação Nacional do Índio - Funai

http://www.funai.gov.br/

Funasa - Fundação Nacional de Saúde

http://www.funasa.gov.br/

Museu do Índio

http://www.museudoindio.org.br/

Renisi - Rede Nacional de Estudos e Pesquisas em Saúde dos Povos Indígenas

https://sis.funasa.gov.br/portal/

\section{Institutions Universitaires et d'autres sources de recherches}

Arqueologia Brasileira

http://www.itaucultural.org.br/arqueologia/

Bay - Universidade Indígena (revue)

http://www.letras.ufmg.br/bay/index.htm

Documenta Indigena/Centro de documentanção em história indígena

http://documenta.incubadora.fapesp.br/portal

Comissão Pró-Yanomami - CCPY

www.proyanomami.org.br

Coordenação das Organizações Indígenas da Amazônia Brasileira - COIAB

www.coiab.com.br

Conselho Indígena de Roraime - CIR

www.cir.org.br

Lali - Laboratório de Línguas Indígenas - IL/UNB

http://www.unb.br/il/lali/

Museu de Arqueologia e Etnologia da USP - MAE/USP

http://www.mae.usp.br/ 
Núcleo de Estudos e Pesquisas das Populações Indígenas - Neppi

http://www.neppi.org/

Núcleo de Estudos e Pesquisas Sobre Etnicidade - Nepe/UFPE

http://www.ufpe.br/nepe/

Centro de estudos Amerindios (Cesta)/USP

http://www.usp.br/cesta/

Núcleo Insikiran de Formação Superior Indígena

http://www.insikiran.ufrr.br/

Núcleo de Transformações Indígenas - Nuti

http://www.nuti.scire.coppe.ufrj.br/

Página do Melatti

http://www.geocities.com/RainForest/Jungle/6885/

Projeto Trilhas do Conhecimento

http://www.laced.mn.ufrj.br/trilhas/trilhas.htm

Terceiro Grau Indígena - Campus de Barra do Bugres

http://www.unemat.br/ indigena/homepage.htm

\section{Sites governamentaux (Amérique Latine et Europe)}

Abya Yala Net

http://abyayala.nativeweb.org

Coica - Coordinadora de lãs Organizaciones Indígenas de la Cuenca Amazônica

http://www.coica.org/

Cultural Survival

www.cs.org

Directorio de Organizaciones Indígenas de Sudamérica

http://www.laneta.apc.org/rci/dirint/sudam.html

Fundación Gaia Amazonas

http://www.gaiaamazonas.org/

GAT - Gente, Ambiente y Território (Paraguai)

http://www.gat.org.py/

IWGIA - International Work Group for Indigenous Affairs

http://www.iwgia.org/

Linda - Lenguas Indígenas de la Amazônia

http://www.abyayala.org/Linda/ 
The Rainforest Foundation - US

http://www.rainforestfoundation.org

\section{NOTES}

1. Cycle de Films qui a eu lieu au Memorial da América Latina, à Sao Paulo en 1990.

2. S'il y a eu une importante augmentation démographique dans la majorité des peuples, il est néanmoins impossible de négliger le fait que certains peuples ont vu leur population diminuer et que d'autres sont toujours menacés d'extinction.

3. Instituto Brasileiro de Geografia e Estatística. http://indigenas.ibge.gov.br/mapasindigenas-2 (Consulté le 5 mars,2014)

4. Au cours de ces 10 dernières années il a néanmoins été acquis qu'un représentant indigène participe du Conseil National de l'Education et que soient adoptés des matériels didactiques incluant des savoirs indigènes,

5. Traduction du passage du livre de l'auteur: “....idas e vindas entre a vida inventada e a vida vivida, dão o tom do filme e serão decisivas para chegarmos à festa - paradigma dessa mistura entre performance e cotidiano, entre memória e fabulação, cosmologia a atualidade. A festa não é algo que existe e será documentada, mas algo que com o filme irá se produzir. Sucedem-se sequências em que os personagens ensaiam, lembram, se corrigem"

6. Voir: Frota, M. (1996: 258-282) et aussi l'étude pionnière sur les médias autochtones de Terence Turner: Defiant Images; the Kayapo Appropriation of Video, Blackwell Editor, 1992.

7. Je vous invite à lire l'analyse de André Brasil (2012) sur le film Bicicletas de Nhanderu, produit par le "Collectif Mbyá-Gurani de Cinema", dont la réalisation a suscité un débat considérable dans la communauté - comme il arrive souvent dans ces groupes. Néanmoins l'aspect singulier à relever est le fait que ce débat soit incorporé dans le film lui-même de façon qu'à la fois le village fait partie du film et le film fait partie du village.

8. La création de Pajé Filmes coïncide avec un atelier audiovisuel proposée par les élèves $\mathrm{du}$ Cours Normal Indigène des Collèges du Programme d'Implantation d'Ecoles Indigènes du Minas Gerais - PIEIMG, implanté dans le Parc Domanial du Rio Doce, dans le Minas Gerais, en 2008. L'axe de Langages Multiples visait à offrir aux élèves indigènes des expériences expressives à travers des langages médiatiques.

9. Lire: "Canal Futura exibe série que apresenta o olhar dos povos indígenas. http:// www.cartamaior.com.br/?/Editoria/Midia/Canal-Futura-exibe-serie-que-apresenta-oolhar-dos-povos-indigenas-/12/12306. (téléchargé par TV Futura, 01.12.2006, accessed 5 March, 2013)

10. Projeto navegar partners. http://www.youtube.com/watch?v=QQe9mmIyON4 (téléchargé par LatinAmerica bureau 28.07.2010, consulté le 5 mars, 2014)

11. Voir: Ecovox piloto, 2012. http://www.youtube.com/watch? $\mathrm{v}=\mathrm{hTpcVUKMBsI \& feature=c4-.overview \& list=LLrsuOTkWND3cPRKQ}$ ASZ26w

(téléchargé par ecovoxtv 16.05.2012, consulté le 5 mars, 2014) 
12. Sur le "cyberindianisme", ou la capacité des peuples autochtones de gérer la transmissions des savoirs dans le cyberespace, je vous invite à lire les textes, fondées sur les réalités au Canada, rassemblés par Florence Dupré et Marie Lachapelle. In Cahiers du CIÉRA, Cyberespaces et médiatisation des cultures", numéro 5, avril, 2010, Université Laval (Québec). À propos des "manières créatives dont différents groupes aborigènes australiens se saisissent d'interfaces en ligne pour présenter des images choisies de leur culture à une audience globale", comme écrit l'auteur tout au début de son article, je souligne l'article de Jessica de Largy Healy, "Yolngu Zorba meets Superman. Australian Aboriginal people, mediated publicness and the culture of sharing on the Internet" In Anthrovision, 1.1, 2013, http://anthrovision.revues.org/96.

\section{ABSTRACTS}

Taking as a starting point the indigenous filmmaking experiences in Brazil, established during the last twenty years, is it possible to outline the dissemination of filmic images from the contemporary Native American perspective. How do these authors in Brazil manage to circulate their productions? To what extent do they appropriate the vehicles/means available on Internet for this purpose? Which are the constraints? Is it possible to talk about an indigenous leadership in this area?

Prenant comme point de départ des expériences filmiques produites depuis une vingtaine d'années par les Amérindiens au Brésil il est possible de suivre les circuits de diffusion de ces images dans une perspective amérindienne contemporaine. Comment ces auteurs fontils circuler leurs productions ? Dans quelle mesure s'approprient-ils les ressources disponibles sur Internet? Quelles sont les contraintes subies? Peut-on parler d'un protagonisme/activisme amérindien dans ce domaine?

Tomando como punto de partida experiencias cinematográficas de los pueblos indígenas en Brasil durante los últimos veinte años, es posible esbozar la difusión de imágenes fílmicas desde una perspectiva indígena contemporánea.¿Cómo estos autores en Brasil hacen circular sus producciones? Hasta qué punto se apropian de los recursos disponibles en Internet para este fin? ¿Cuáles son las dificultades? ¿Es posible hablar de un protagonismo indígena en este área?

\section{INDEX}

Mots-clés: cinéma amérindien, vidéo amérindienne, Brésil, cyberspace, nouvelles technologies Palabras claves: cine indígena, video indígena, Brasil, ciberespacio, nuevas tecnologías Keywords: Native cinema, indigenous video, Brazil, cyberspace, new technologies 


\section{AUTHOR}

\section{PAULA MORGADO}

Université de Sao Paulo

Laboratoire d'Image et Son en Anthropologie/LISA

lopes@usp.br 